\title{
The effects of environmental hypoxia on substrate utilisation during exercise: a meta-analysis
}

\author{
Alex Griffiths ${ }^{1 *}$ (D), Oliver M. Shannon ${ }^{1,2}$, Jamie Matu ${ }^{1,3}$, Roderick King ${ }^{1}$, Kevin Deighton ${ }^{1}$ and John P. O'Hara ${ }^{1}$
}

\begin{abstract}
Background: A better understanding of hypoxia-induced changes in substrate utilisation can facilitate the development of nutritional strategies for mountaineers, military personnel and athletes during exposure to altitude. However, reported metabolic responses are currently divergent. As such, this systematic review and meta-analysis aims to determine the changes in substrate utilisation during exercise in hypoxia compared with normoxia and identify study characteristics responsible for the heterogeneity in findings.

Methods: A total of six databases (PubMed, the Cochrane Library, MEDLINE, SPORTDiscus, PsychINFO, and CINAHL via EBSCOhost) were searched for published original studies, conference proceedings, abstracts, dissertations and theses. Studies were included if they evaluated respiratory exchange ratio (RER) and/or carbohydrate or fat oxidation during steady state exercise matched for relative intensities in normoxia and hypoxia (normobaric or hypobaric). A random-effects meta-analysis was performed on outcome variables. Meta-regression analysis was performed to investigate potential sources of heterogeneity.

Results: In total, 18 studies were included in the meta-analysis. There was no significant change in RER during exercise matched for relative exercise intensities in hypoxia, compared with normoxia (mean difference: 0.01, 95\% Cl: -0.02 to $0.05 ; n=31, p=0.45$ ). Meta-regression analysis suggests that consumption of a pre-exercise meal $(p<0.01)$ and a higher exercise intensity $(p=0.04)$ when exposed to hypoxia may increase carbohydrate oxidation compared with normoxia.

Conclusions: Exposure to hypoxia did not induce a consistent change in the relative contribution of carbohydrate or fat to the total energy yield during exercise matched for relative intensities, compared with normoxia. The direction of these responses appears to be mediated by the consumption of a pre-exercise meal and exercise intensity.
\end{abstract}

Keywords: Altitude, Exercise, Substrate, Carbohydrate, Fat, Oxidation, Systematic review

\section{Background}

An increasing number of people ascend to altitude each year for recreational, occupational, and sporting purposes [1]. The hypoxic exposure experienced at altitude is known to cause a curvilinear impairment in endurance performance with increasing levels of hypoxia [2]. However, the changes in substrate utilisation associated with these decrements in performance are currently unclear, with some authors reporting an increased

\footnotetext{
*Correspondence: A.Griffiths@leedsbeckett.ac.uk

${ }^{1}$ Institute for Sport, Physical Activity and Leisure, Leeds Beckett University, Leeds LS6 3QS, UK

Full list of author information is available at the end of the article
}

contribution of carbohydrate to the total energy yield [3, 4], and others demonstrating an increased contribution of fat oxidation [5, 6]. Developing a better understanding of these changes in substrate utilisation in hypoxia is vital in designing dietary interventions to maintain and/ or improve performance in such conditions.

Exposure to hypoxic environments may alter substrate utilisation through multifarious mechanisms. It is purported that the mechanism responsible for increased carbohydrate dependency in hypoxia is mediated by the sympathetic nervous system, via the secretion of epinephrine and norepinephrine, stimulating glycogenolysis and gluconeogenesis [3, 7]. Alternative evidence suggests

(C) The Author(s). 2019 Open Access This article is distributed under the terms of the Creative Commons Attribution 4.0 International License (http://creativecommons.org/licenses/by/4.0/), which permits unrestricted use, distribution, and 
that increases in the transcription factor hypoxic-inducible factor 1 alpha (HIF-1 $\alpha$ ) may be responsible for the increases in fat oxidation observed by some in hypoxia, via upregulation of the fatty acid-activated transcription factor peroxisome proliferator-activated receptor alpha (PPAR $\alpha)$ [8]. Albeit in rats, PPAR $\alpha$ has been demonstrated to deactivate pyruvate dehydrogenase [9], inhibiting the conversion of pyruvate to acetyl-coA and therefore enabling greater fat flux for oxidation [10].

A myriad of factors has been suggested to influence the interaction between hypoxic exposure and substrate utilisation. These include, but are not limited to: characteristics of hypoxic exposure (severity, duration and type) [3], nutritional status of participants (fasted or fed before exercise/exogenous supplementation during exercise) $[4,5]$ and sex of participants [11]. In relation to hypoxic exposure, hypobaric hypoxia $(\mathrm{HH})$ has been suggested to elicit more severe physiological responses (greater hypoxemia and lower arterial oxygen saturation) compared with normobaric hypoxia (NH) [12]. Although this is contested in the literature $[13,14]$ it seems plausible that these physiological differences may induce a greater reliance on carbohydrate oxidation to achieve a higher yield of ATP per unit of oxygen consumption, compared with fat oxidation [15]. This theory may also be applied to the effect of altitude severity on subsequent substrate oxidation. Further, metabolic responses may be different between sex, with females demonstrating a greater relative utilisation of fat oxidation $[11,16]$. This may be attributable to a number of factors, with females demonstrating a greater relative fat mass [17] and intramuscular triglyceride stores [18], as well as better mobilisation of free fatty acid (FFA) from subcutaneous adipose tissue [19]. It has also been suggested that this propensity for fat oxidation may be mediated by the steroid hormones estrogen (predominantly $17 \quad \beta$-estradiol) and progesterone [20,21]. Regarding nutritional status of participants, equivocal metabolic findings have been observed in response to carbohydrate supplementation during exercise in hypoxia, compared with normoxia $[4,5]$, which demonstrates the limited current understanding of the interaction between dietary interventions and hypoxic exposure.

To gain a clear understanding of changes in substrate utilisation during exercise in hypoxia compared with normoxia, a systematic evaluation is required to explain the equivocal results of previous studies. Due to the greater exercise-induced physiological stress experienced when performing a matched absolute workload under hypoxic conditions [22], this meta-analysis focuses solely on exercise matched to relative intensities. The aim of this meta-analysis was to identify the study characteristics responsible for heterogeneity between findings, using subgroup analyses and meta-regression.

\section{Methods}

The current systematic review and meta-analysis was performed in accordance with the Preferred Reporting Items for Systematic Review and Meta-analyses (PRISMA) guidelines [23].

\section{Literature search}

A literature search was conducted using the electronic bibliographic databases PubMed and the Cochrane Library, as well as searching MEDLINE, SPORTDiscus, PsychINFO, and CINAHL via EBSCOhost. The initial search of titles, abstracts and keywords was conducted on 15th November 2016 using terms related to 'exercise', 'hypoxia,' 'substrate' and 'oxidation'. A final search was conducted on 5th June 2018. The specific keywords and full search strategy can be found in Additional file 1 . The reference lists of all included studies and relevant review articles were screened for possible inclusion. No language restrictions were applied and in the case of studies available only as an abstract, authors were contacted for the full dataset.

\section{Inclusion criteria}

Included studies were required to meet the following criteria: participants in the study were between the ages of 18 and 65 years, not pregnant, non-smokers, with no history of diabetes, gastrointestinal, inflammatory, metabolic, cardiovascular, neurological or psychological disease. In order to minimise potential publication bias, studies published in peer reviewed journals, conference proceedings, theses or dissertations were eligible for inclusion.

All studies were required to measure RER and/or carbohydrate or fat oxidation. These measures were required to be quantified during exercise matched for relative intensities in hypoxic and normoxic environments. Hypoxic exposure was defined as terrestrial altitude via geographical location (TA) or simulated altitude $(\mathrm{NH}$ or $\mathrm{HH}$ ) via a hypoxic tent, hypoxic chamber or breathing mask. Exposures were required to be $>1500 \mathrm{~m}$ or a simulated equivalent (i.e., low altitude or higher) [24]. All participants within selected studies had not been exposed to $>1500 \mathrm{~m}$ (or a simulated equivalent) within the previous 3 months. Normoxic trials were required to provide a viable within-participant control (i.e. equivalent measure(s) quantified in the same participants as a separate trial in normoxic conditions). The exercise was required to be $>5 \mathrm{~min}$ in duration to achieve steady-state values at a fixed exercise intensity [25].

Two researchers (AG and OS) independently assessed studies for inclusion and later compared notes to reach a mutual consensus. Disagreements about the eligibility of any particular studies were resolved by a third reviewer (KD). Potential studies that could not be excluded 
based on their title or abstract were retrieved in full-text and reviewed against the inclusion/exclusion criteria independently by two researchers (AG and OS) with a third researcher (KD) used to settle any disputes. In total, 18 studies met the inclusion criteria and were included in this meta-analysis.

\section{Abstraction of data}

Data were extracted independently by two researchers (AG and OS) into a standardised spreadsheet, which included (i) characteristics of articles valid for review; (ii) the Cochrane Collaboration's tool for assessing risk of bias, and (iii) outcome data suitable for analysis based on mean, standard deviation (SD) and sample size. Further data was extracted regarding participant characteristics, acclimatisation status, nutritional manipulations, exercise intensities and duration, exercise mode, and severity and duration of hypoxic exposure. In studies which employed multiple exercise intensities, each respective intensity was directly compared with the equivalent intensity in the alternate condition.

In studies which reported outcome variables across numerous time points during exercise, values were averaged to calculate the mean. In addition, SD values were averaged using the following formula:

$$
\frac{\mathrm{n}^{1}\left(\mathrm{~S} 1^{2}+\mathrm{D} 1^{2}\right)+\mathrm{n}^{2}\left(\mathrm{~S} 2^{2}+\mathrm{D} 2^{2}\right) \ldots}{\left(\mathrm{n}^{1}+\mathrm{n}^{2}\right) \ldots}
$$

where:

$$
\begin{aligned}
& \mathrm{n}^{1}=\text { sample size of group } 1 \\
& \mathrm{n}^{2}=\text { sample size of group } 2 \\
& \mathrm{~S} 1=\text { SD of group } 1 \\
& \mathrm{~S} 2=\mathrm{SD} \text { of group } 2 \\
& \mathrm{D} 1=\text { mean of group } 1-\text { mean of total group } \\
& \mathrm{D} 2=\text { mean of group } 2-\text { mean of total group } \\
& \ldots \text { denotes inclusion of further data points if required }
\end{aligned}
$$

Absolute substrate oxidation data was converted to g. $\mathrm{min}^{-1}$. Thus, values expressed as total grams oxidised throughout exercise were divided by the number of minutes the variable was measured. Values expressed in $\mathrm{mg} \cdot \mathrm{kcal} \cdot \mathrm{min}^{-1}$ were multiplied by 1000 to convert to $\mathrm{g} \cdot \mathrm{kcal} \cdot \mathrm{min}^{-1}$, and then multiplied by $\mathrm{kcal}$ values provided in the relevant paper for the conversion to g. $\mathrm{min}^{-}$ ${ }^{1}$. In addition, carbohydrate oxidation data provided in $\mathrm{mmol} \cdot \mathrm{min}^{-1}$ were divided by 1000 and then multiplied by the molar mass of glucose $(180.1559 \mathrm{~g} / \mathrm{mol})$. Where values were presented as figures, these were digitized using graph digitizer software (DigitizeIt, Germany) and the means and SD were measured manually at the pixel level to the scale provided on the figure.

\section{Assessment of risk of bias in included studies}

Two independent reviewers (AG and OS) used The Cochrane Collaboration's tool for assessing risk of bias [26] to determine the risk of bias in each study. Each study was assessed in the following six domains: sequence generation, allocation concealment, blinding of participants, personnel and outcome assessors, incomplete outcome data, selective outcome reporting and other sources of bias (e.g. has been claimed to have been fraudulent). A judgement was made on each of the domains by the two independent researchers as to whether they were 'high risk 'or 'low risk'. When insufficient detail was reported then the judgement of 'unclear risk' was made. Disagreements were solved initially via discussion between the two independent reviewers however a third reviewer (KD) was consulted for dispute resolution. 'Risk of bias graphs' were computed in Review Manager (RevMan) 5.3 (The Cochrane Collaboration) to include low, unclear and high risk for each domain.

\section{Statistical analysis}

Outcome measures were quantified using mean difference between conditions with 95\% confidence intervals (CI) which were used as the summary statistic. A random-effects meta-analysis was performed by AG, JM and KD using Comprehensive Meta-Analysis Software (version 3, Biostat, Englewood, NJ, USA). The inputted data included sample sizes, outcome measures with their respective SDs, and a correlation coefficient for within-participant measurements. These correlation coefficients were estimated from prior studies in our laboratory and were as follows: RER $r=0.78$, absolute carbohydrate oxidation $r=0.70$, absolute fat oxidation $r=0.81$, relative carbohydrate oxidation $r=0.79$, relative fat oxidation $r=0.79[5,6]$.

A negative mean difference indicates that hypoxic exposure was associated with a decrease in the respective outcome variable, while a positive mean difference indicates that hypoxic exposure was associated with an increase in the respective outcome variable. Heterogeneity between trials was assessed using the Chi-squared statistic, $I$-squared statistic and the Tau-squared statistic.

To examine whether any conclusions were dependent on a single study, sensitivity analyses was employed for each variable by repeating the analyses with each study omitted in turn.

Where the number of comparisons was suitable, meta-regression analysis was performed. This analysis was used to determine whether continuous or categorical data, including severity of hypoxic exposure, exercise intensity and pre-exercise nutritional state could explain the variation in the values observed between studies. Each moderator was analysed in a meta-regression independently to determine their relationship with the outcome variable. Where significant moderators were identified, multiple meta-regression analysis was conducted to determine if 
these remained significant, whilst controlling for all other moderators. All meta-regressions were performed using the restricted maximum likelihood (REML) method with Knapp-Hartung adjustment.

Duration of hypoxic exposure was categorised into acute and chronic as per each study's description of their own exposure (acute $<44 \mathrm{~h}$; chronic $=3-28$ days). Overall mean differences, CIs and $p$ values were reported for all variables during exercise matched to relative intensities. Meta-regression analysis was performed only on the outcome variable RER, as this represents the most appropriate measure to assess changes in the relative substrate contributions of carbohydrate and fat (i.e., physiological shifts in substrate utilisation). Meta-regression analysis of absolute fat and carbohydrate oxidation rates was deemed unnecessary based on these responses determining the RER values for each study.

\section{Exploration of small study effects}

Small study effects were explored using funnel plots of mean difference versus standard errors [26], and by quantifying Egger's linear regression intercept. A statistically significant Egger's statistic $(p<0.05)$ indicates the presence of small study effects.

\section{Results}

\section{Overview}

A total of 1743 studies published in peer reviewed scientific journals were initially identified through database screening and other sources. Following the full screening process, 18 studies were identified as suitable for the meta-analyses (Fig. 1). Within the 18 studies, a total of 58 comparisons between normoxic and hypoxic conditions were made for exercise matched for relative intensities. Of these 58 comparisons, 31 reported RER (Table 1) and 27 reported substrate utilisation (Table 2) ((absolute carbohydrate oxidation $=7$, absolute fat oxidation $=6$; relative carbohydrate oxidation $=7$, relative fat oxidation $=7$ ).

\section{Participant demographics and study characteristics}

Of the 170 participants included in the analysis, 146 were male $(86 \%)$ and 24 were female $(14 \%)$. Age was reported in all studies and ranged from 20 to 39 years (mean $=27$ years). BMI was reported in 15 of the 18 studies and ranged from 21.3 to $28.6 \mathrm{~kg} \cdot \mathrm{m}^{-2}\left(\right.$ mean $\left.=23.4 \mathrm{~kg} \cdot \mathrm{m}^{-2}\right)$. $\mathrm{VO}_{2 \max }$ was reported in 17 of the 18 studies and ranged between 2.61 and $4.99 \mathrm{~L} \cdot \mathrm{min}^{-1}\left(\right.$ mean $\left.=3.75 \mathrm{~L} \cdot \mathrm{min}^{-1}\right)$.

Exercise duration ranged from 5 to 105 min (mean = $39 \mathrm{~min}$ ). Participants in normoxic trials performed exercise at intensities ranging from 30 to $82 \%$ of normoxic $\mathrm{VO}_{2 \max }\left(\right.$ mean $\left.=61 \% \mathrm{SL} \mathrm{VO}_{2 \max }\right)$ and hypoxic trials were performed at $30-83 \%$ of hypoxic specific $\mathrm{VO}_{2 \max }$ (mean $=61 \%$ hypoxic $\mathrm{VO}_{2 \max }$ ). The severity of hypoxia quantified in meters, ranged from $1500 \mathrm{~m}$ to $4300 \mathrm{~m}$ (mean =
$3499 \mathrm{~m}$ ). Feeding status was only specified in 26 out of 31 comparisons (fasted $=8$; fed $=18$ ).

\section{Meta-analysis}

Individual study statistics and results for each variable are provided in the supplementary tables (Additional files 2, 3, 4,5 and 6 ).

\section{RER}

There was no significant change in RER during exercise matched for relative intensities in hypoxia, compared with normoxia (mean difference: $0.01,95 \%$ CI: -0.02 to $0.05 ; n=31, p=0.45$; Fig. 2). The degree of heterogeneity was found to be high between studies $\left(I^{2}=99.87 \%, \mathrm{Q}=\right.$ $\left.27,768, \tau^{2}=0.01, d_{f}=30\right)$. Sensitivity analysis revealed minor changes only, and these changes did not substantially alter the overall mean difference. Inspection of the funnel plot and Egger's regression intercept revealed that there was little evidence of small study effects (intercept $=12.61,95 \%$ CI: -5.87 to $31.08 ; p=0.17$ ).

Meta-regression analysis (Table 3) indicated a difference in RER responses between participants in the fasted and fed state $(p<0.01)$, with participants in the fasted state demonstrating a decreased RER, and those in the fed state demonstrating an increased RER during exercise matched for relative exercise intensity in hypoxia, compared with normoxia. Using exercise intensity as a moderator, a greater exercise intensity was associated with a greater increase in RER during exercise matched to relative intensity in hypoxia, compared with normoxia. The slope of the regression was significantly positive $(p=0.04)$, with a standardised increase of 0.0033 units, for every percentage increase in exercise intensity. When the significant variables from the bivariate analysis were entered into the multiple regression models, both pre-exercise nutritional state and exercise intensity remained significant. The multiple regression model explained $42 \%$ of the variance observed $\left(R^{2}=0.42\right)$.

\section{Relative carbohydrate and fat oxidation rates}

There was no significant change in relative carbohydrate oxidation rates during exercise matched for relative intensities in hypoxia, compared with normoxia (mean difference: $1.74,95 \%$ CI: -4.76 to $8.25 \% ; n=7, p=0.60$; Additional file 7). The degree of heterogeneity was found to be high between studies $\left(I^{2}=99.09 \%, Q=659, \tau^{2}=\right.$ $71.00, d_{f}=6$ ). Sensitivity analysis revealed minor changes only, and these changes did not substantially alter the overall mean difference. Inspection of the funnel plot and Egger's regression intercept revealed that there was little evidence of small study effects (intercept $=0.69$, 95\% CI: -16.79 to $18.17 ; p=0.92$ ).

There was no significant change in relative fat oxidation during exercise matched for relative intensities in 


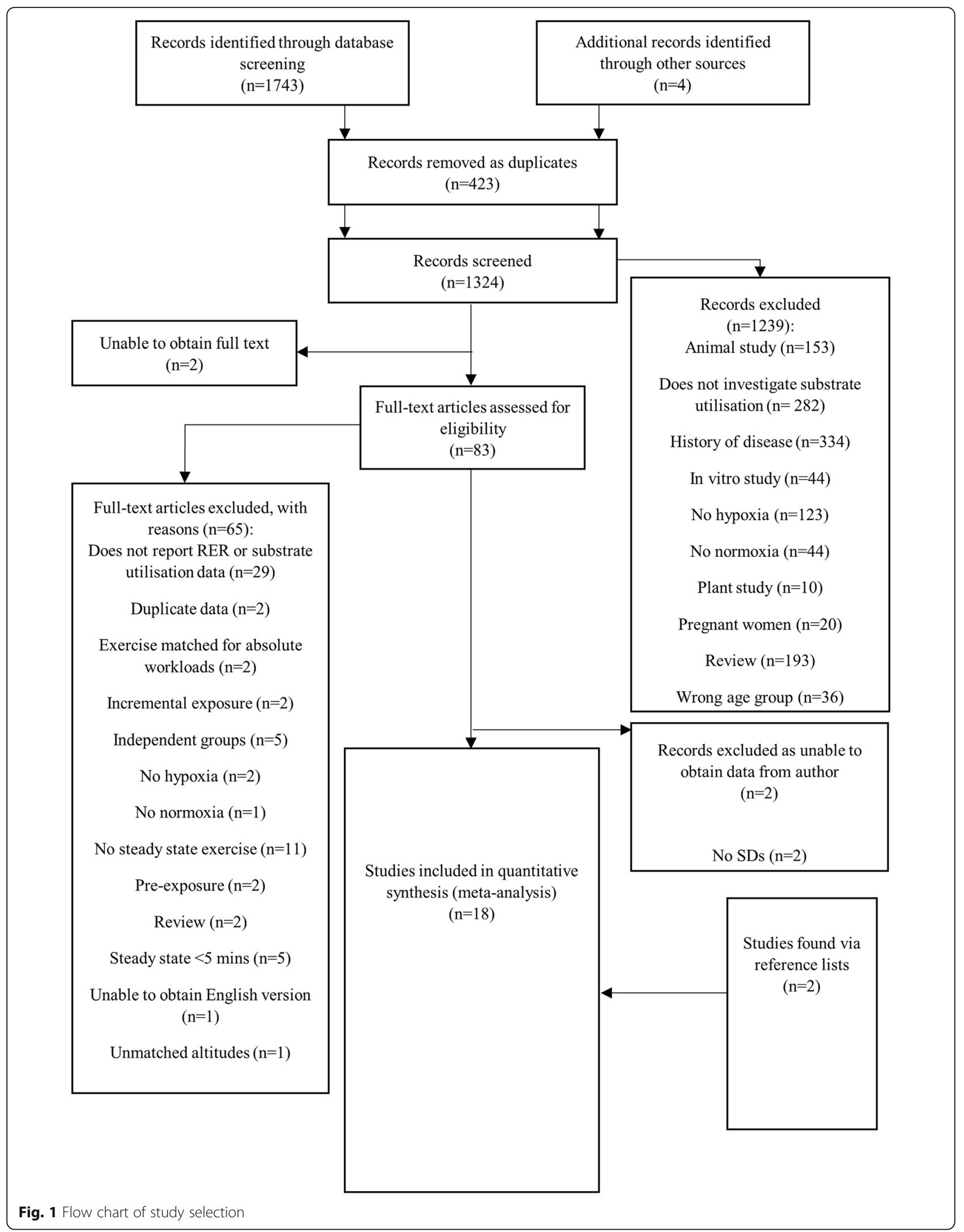


Table 1 Studies investigating RER during exercise matched for relative intensities in hypoxia, compared with normoxia

\begin{tabular}{|c|c|c|c|c|c|c|}
\hline Study & Participants & Study design & $\begin{array}{l}\text { Type of } \\
\text { hypoxia }\end{array}$ & Altitude $(\mathrm{m})$ & $\begin{array}{l}\text { Duration } \\
\text { of hypoxia }\end{array}$ & RER \\
\hline $\begin{array}{l}\text { Beidleman et al. } \\
\text { (2002) [16] }\end{array}$ & 8 (females) & $\begin{array}{l}\text { Treadmill exercise at } 70 \% \text { relative } \\
\mathrm{VO}_{2 \max } \text { until volitional exhaustion }\end{array}$ & $\mathrm{HH}$ & 4300 & $180 \mathrm{~min}$ & $\begin{array}{l}\text { SL: } 0.89 \pm 0.04 \\
\text { AH: } 0.85 \pm 0.03\end{array}$ \\
\hline $\begin{array}{l}\text { Beidleman et al. } \\
\text { (2003) A [45] }\end{array}$ & $\begin{array}{l}6(\text { male }=5 \\
\text { female }=1)\end{array}$ & 15 min cycling at $40 \%$ relative $\mathrm{VO}_{2 \max }$ & $\mathrm{HH}$ & 4300 & $70 \mathrm{~min}$ & $\begin{array}{l}\text { SL: } 0.97 \pm 0.02 \\
\text { AH: } 0.88 \pm 0.01\end{array}$ \\
\hline $\begin{array}{l}\text { Beidleman et al. } \\
\text { (2003) B [45] }\end{array}$ & $\begin{array}{l}6(\text { male }=5 \\
\text { female }=1)\end{array}$ & 15 min cycling at $70 \%$ relative $\mathrm{VO}_{2 \max }$ & $\mathrm{HH}$ & 4300 & $70 \mathrm{~min}$ & $\begin{array}{l}\text { SL: } 1.02 \pm 0.02 \\
\text { AH: } 1.00 \pm 0.03\end{array}$ \\
\hline $\begin{array}{l}\text { Bouissou et al. } \\
\text { (1987) [46] }\end{array}$ & 6 (males) & 60 min cycling at $60 \%$ relative $\mathrm{VO}_{2 \max }$ & $\mathrm{HH}$ & 3000 & $80 \mathrm{~min}$ & $\begin{array}{l}\text { SL: } 0.99 \pm 0.02 \\
\text { AH: } 1.00 \pm 0.01\end{array}$ \\
\hline Braun et al. (2000) [11] & 15 (females) & 30 min cycling at $65 \%$ relative $\mathrm{VO}_{2 \max }$ & TA & 4300 & 10 days & $\begin{array}{l}\text { SL: } 0.97 \pm 0.01 \\
\text { CH: } 0.94 \pm 0.01\end{array}$ \\
\hline $\begin{array}{l}\text { Friedmann et al. } \\
\text { (2004) [47] }\end{array}$ & 11 (males) & 60 min running at $\sim 82 \%$ relative $\mathrm{VO}_{2 \max }$ & $\mathrm{NH}$ & 2500 & $120 \mathrm{~min}$ & $\begin{array}{l}\text { SL: } 0.93 \pm 0.02 \\
\text { AH: } 0.98 \pm 0.07\end{array}$ \\
\hline $\begin{array}{l}\text { Fulco et al. (2005) } \\
\text { A [48] }\end{array}$ & 16 (males) & 20 min cycling at $48 \%$ relative $\mathrm{VO}_{2 \max }$ & TA & 4300 & 3 days & $\begin{array}{l}\text { SL: } 0.91 \pm 0.01 \\
\text { CH: } 0.81 \pm 0.01\end{array}$ \\
\hline $\begin{array}{l}\text { Fulco et al. (2005) } \\
\text { B [48] }\end{array}$ & 16 (males) & 20 min cycling at $48 \%$ relative $\mathrm{VO}_{2 \max }$ & TA & 4300 & 10 days & $\begin{array}{l}\text { SL: } 0.91 \pm 0.01 \\
\text { CH: } 0.80 \pm 0.01\end{array}$ \\
\hline $\begin{array}{l}\text { Fulco et al. (2005) } \\
\text { C [48] }\end{array}$ & 16 (males) & 20 min cycling at $68 \%$ relative $\mathrm{VO}_{2 \max }$ & TA & 4300 & 3 days & $\begin{array}{l}\text { SL: } 0.93 \pm 0.01 \\
\text { CH: } 0.85 \pm 0.01\end{array}$ \\
\hline $\begin{array}{l}\text { Fulco et al. (2005) } \\
\text { D [48] }\end{array}$ & 16 (males) & 20 min cycling at $68 \%$ relative $\mathrm{VO}_{2 \max }$ & TA & 4300 & 10 days & $\begin{array}{l}\text { SL: } 0.93 \pm 0.01 \\
\text { CH: } 0.84 \pm 0.01\end{array}$ \\
\hline $\begin{array}{l}\text { Hopkins et al. (2003) } \\
\text { A [49] }\end{array}$ & $\begin{array}{l}6(\text { male }=1 \\
\text { female }=5)\end{array}$ & 5 min cycling at $30 \%$ relative $\mathrm{VO}_{2 \max }$ & $\mathrm{NH}$ & 3850 & $25 \mathrm{~min}$ & $\begin{array}{l}\text { SL: } 0.82 \pm 0.02 \\
\text { AH: } 0.86 \pm 0.03\end{array}$ \\
\hline $\begin{array}{l}\text { Hopkins et al. (2003) } \\
\text { B [49] }\end{array}$ & $\begin{array}{l}6(\text { male }=1 \\
\text { female }=5)\end{array}$ & 5 min cycling at $60 \%$ relative $\mathrm{VO}_{2 \max }$ & $\mathrm{NH}$ & 3850 & $25 \mathrm{~min}$ & $\begin{array}{l}\text { SL: } 0.95 \pm 0.02 \\
\text { AH: } 0.96 \pm 0.03\end{array}$ \\
\hline $\begin{array}{l}\text { Katayama et al. } \\
\text { (2010) [3] }\end{array}$ & 7 (males) & 30 min cycling at $50 \%$ relative $\mathrm{VO}_{2 \max }$ & $\mathrm{HH}$ & 2000 & $100 \mathrm{~min}$ & $\begin{array}{l}\text { SL: } 0.90 \pm 0.01 \\
\text { AH: } 0.91 \pm 0.02\end{array}$ \\
\hline Lundby and Van Hall (2002) A [22] & $\begin{array}{l}8(\text { male }=6, \\
\text { female }=2)\end{array}$ & 60 min cycling at $50 \%$ relative $\mathrm{VO}_{2 \max }$ & $\mathrm{NH}$ & 4100 & $70 \mathrm{~min}$ & $\begin{array}{l}\text { SL: } 0.91 \pm 0.01 \\
\text { AH: } 0.92 \pm 0.02\end{array}$ \\
\hline $\begin{array}{l}\text { Lundby and Van Hall } \\
\text { (2002) B [22] }\end{array}$ & $\begin{array}{l}8(\text { male }=6, \\
\text { female }=2)\end{array}$ & 60 min cycling at $50 \%$ relative $\mathrm{VO}_{2 \max }$ & TA & 4100 & 28 days & $\begin{array}{l}\text { SL: } 0.91 \pm 0.01 \\
\text { CH: } 0.91 \pm 0.01\end{array}$ \\
\hline $\begin{array}{l}\text { Maher et al. (1974) } \\
\text { A [50] }\end{array}$ & 8 (males) & 10 min cycling at $75 \%$ relative $\mathrm{VO}_{2 \max }$ & TA & 4300 & $44 \mathrm{~h}$ & $\begin{array}{l}\text { SL: } 0.99 \pm 0.01 \\
\text { AH: } 0.98 \pm 0.01\end{array}$ \\
\hline $\begin{array}{l}\text { Maher et al. (1974) } \\
\text { B [50] }\end{array}$ & 8 (males) & 10 min cycling at $75 \%$ relative $\mathrm{VO}_{2 \max }$ & TA & 4300 & 12 days & $\begin{array}{l}\text { SL: } 0.99 \pm 0.01 \\
\text { CH: } 0.98 \pm 0.01\end{array}$ \\
\hline Matu et al. (2017) A [6] & 12 (males) & 60 min walking at $50 \%$ relative $\mathrm{VO}_{2 \max }$ & $\mathrm{NH}$ & 2150 & $5 \mathrm{~h}$ & $\begin{array}{l}\text { SL: } 0.88 \pm 0.04 \\
\mathrm{AH}: 0.85 \pm 0.06\end{array}$ \\
\hline Matu et al. (2017) B [6] & 12 (males) & 60 min walking at $50 \%$ relative $\mathrm{VO}_{2 \max }$ & $\mathrm{NH}$ & 4300 & $5 \mathrm{~h}$ & $\begin{array}{l}\text { SL: } 0.88 \pm 0.04 \\
\text { AH: } 0.85 \pm 0.06\end{array}$ \\
\hline Messier et al. (2017) [51] & 20 (males) & 60 min cycling at $\sim 67 \%$ relative $\mathrm{VO}_{2 \max }$ & TA & 2150 & $150 \mathrm{~min}$ & $\begin{array}{l}\text { SL: } 0.92 \pm 0.06 \\
\text { AH: } 0.97 \pm 0.05\end{array}$ \\
\hline $\begin{array}{l}\text { Noordhof et al. (2013) } \\
\text { A [52] }\end{array}$ & 16 (male) & 6 min cycling at $45 \%$ relative $\mathrm{VO}_{2 \max }$ & $\mathrm{HH}$ & 1500 & $30 \mathrm{~min}$ & $\begin{array}{l}\text { SL: } 0.89 \pm 0.03 \\
\text { AH: } 0.92 \pm 0.02\end{array}$ \\
\hline $\begin{array}{l}\text { Noordhof et al. (2013) } \\
\text { B [52] }\end{array}$ & 16 (male) & 6 min cycling at $55 \%$ relative $\mathrm{VO}_{2 \max }$ & $\mathrm{HH}$ & 1500 & $30 \mathrm{~min}$ & $\begin{array}{l}\mathrm{SL}: 0.91 \pm 0.04 \\
\mathrm{AH}: 0.94 \pm 0.04\end{array}$ \\
\hline $\begin{array}{l}\text { Noordhof et al. (2013) } \\
\text { C [52] }\end{array}$ & 16 (male) & 6 min cycling at $65 \%$ relative $\mathrm{VO}_{2 \max }$ & $\mathrm{HH}$ & 1500 & $30 \mathrm{~min}$ & $\begin{array}{l}\text { SL: } 0.94 \pm 0.04 \\
\text { AH: } 0.98 \pm 0.04\end{array}$ \\
\hline O'Hara et al. (2017) A [5] & 7 (males) & 5 min cycling at $\sim 62 \%$ relative $\mathrm{VO}_{2 \max }$ & $\mathrm{HH}$ & 3375 & 155 min & $\begin{array}{l}\text { SL: } 0.92 \pm 0.04 \\
\text { AH: } 0.83 \pm 0.04\end{array}$ \\
\hline O'Hara et al. (2017) B [5] & 7 (males) & 105 min cycling at $\sim 74 \%$ relative $\mathrm{VO}_{2 \max }$ & $\mathrm{HH}$ & 3375 & $155 \min$ & $\begin{array}{l}\text { SL: } 0.92 \pm 0.03 \\
\text { AH: } 0.84 \pm 0.05\end{array}$ \\
\hline Peronnet et al. (2006) [4] & 5 (males) & 80 min cycling at $77 \%$ relative $\mathrm{VO}_{2 \max }$ & $\mathrm{HH}$ & 4300 & $110 \mathrm{~min}$ & $\begin{array}{l}\text { SL: } 0.93 \pm 0.01 \\
\mathrm{AH}: 0.97 \pm 0.01\end{array}$ \\
\hline
\end{tabular}


Table 1 Studies investigating RER during exercise matched for relative intensities in hypoxia, compared with normoxia (Continued)

\begin{tabular}{|c|c|c|c|c|c|c|}
\hline Study & Participants & Study design & $\begin{array}{l}\text { Type of } \\
\text { hypoxia }\end{array}$ & Altitude $(\mathrm{m})$ & $\begin{array}{l}\text { Duration } \\
\text { of hypoxia }\end{array}$ & RER \\
\hline Wyss et al. (1990) [53] & 7 (males) & 30 min running at $\sim 79 \%$ relative $\mathrm{VO}_{2 \max }$ & $\mathrm{NH}$ & 3500 & $60 \mathrm{~min}$ & $\begin{array}{l}\text { SL: } 0.90 \pm 0.04 \\
\text { AH: } 0.93 \pm 0.04\end{array}$ \\
\hline Young et al. (1987) A [54] & 12 (males) & $\begin{array}{l}30 \text { min cycling at } 75 \% \text { relative } \\
\mathrm{VO}_{2 \max } \text { (active between exercise tests) }\end{array}$ & TA & 4300 & $<24 \mathrm{~h}$ & $\begin{array}{l}\text { SL: } 0.84 \pm 0.02 \\
\text { AH: } 1.03 \pm 0.01\end{array}$ \\
\hline Young et al. (1987) B [54] & 12 (males) & $\begin{array}{l}30 \text { min cycling at } 75 \% \text { relative } \\
\mathrm{VO}_{2 \max } \text { (active between exercise tests) }\end{array}$ & TA & 4300 & 13 days & $\begin{array}{l}\text { SL: } 0.84 \pm 0.02 \\
\text { CH: } 1.03 \pm 0.06\end{array}$ \\
\hline Young et al. (1987) C [54] & 12 (males) & $\begin{array}{l}30 \text { min cycling at } 75 \% \text { relative } \\
\mathrm{VO}_{2 \max } \text { (sedentary between exercise tests) }\end{array}$ & TA & 4300 & $<24 \mathrm{~h}$ & $\begin{array}{l}\text { SL: } 0.84 \pm 0.01 \\
\text { AH: } 1.05 \pm 0.01\end{array}$ \\
\hline Young et al. (1987) D [54] & 12 (males) & $\begin{array}{l}30 \text { min cycling at } 75 \% \text { relative } \\
\mathrm{VO}_{2 \max } \text { (sedentary between exercise tests) }\end{array}$ & TA & 4300 & 13 days & $\begin{array}{l}\text { SL: } 0.84 \pm 0.01 \\
\text { CH: } 1.13 \pm 0.05\end{array}$ \\
\hline
\end{tabular}

Values presented as mean \pm SD. $H H$ hypobaric hypoxia, $N H$ normobaric hypoxia, $T A$ terrestrial altitude, $S L$ sea level, $A H$ acute hypoxia, $C H$ chronic hypoxia. A, B, C and $\mathrm{D}$ refer to the different trial arms of each study

hypoxia, compared with normoxia (mean difference: $-1.74,95 \% \mathrm{CI}=-8.25$ to $4.76 \%, n=7, p=0.60$; Additional file 8). The degree of heterogeneity was found to be high between studies $\left(I^{2}=99.09 \%, Q=659, \mathrm{\tau}^{2}=71.00\right.$, $\left.\mathrm{d}_{\mathrm{f}}=6\right)$. Sensitivity analysis revealed minor changes only, and these changes did not substantially alter the overall mean difference. Inspection of the funnel plot and Egger's regression intercept revealed that there was little evidence of small study effects (intercept $=-0.69,95 \%$ CI: -18.17 to $16.79 ; p=0.92$ ).

\section{Absolute carbohydrate and fat oxidation rates}

There was a significant decrease in absolute carbohydrate oxidation rates during exercise matched for relative intensities in hypoxia, compared with normoxia (mean difference: $-0.57 \mathrm{~g} \cdot \mathrm{min}^{-1}$, $95 \% \mathrm{CI}:-0.74$ to -0.40 g. $\min ^{-1} ; n=7 ; p<0.01 ;$ Fig. 3). The degree of heterogeneity was found to be high between studies $\left(I^{2}=94.66 \%\right.$, $\left.\mathrm{Q}=112, \mathrm{~T}^{2}=0.05, \mathrm{~d}_{\mathrm{f}}=6\right)$. Sensitivity analysis revealed minor changes only, and these changes did not substantially alter the overall mean difference. Inspection of the

Table 2 Studies investigating substrate utilisation during exercise matched for relative intensities in hypoxia compared with normoxia

\begin{tabular}{|c|c|c|c|c|c|c|c|c|c|}
\hline \multirow[t]{2}{*}{ Study } & \multirow[t]{2}{*}{ Participants } & \multirow[t]{2}{*}{ Study design } & \multirow[t]{2}{*}{$\begin{array}{l}\text { Type of } \\
\text { hypoxia }\end{array}$} & \multirow[t]{2}{*}{ Altitude (m) } & \multirow[t]{2}{*}{$\begin{array}{l}\text { Duration } \\
\text { of exposure }\end{array}$} & \multicolumn{2}{|c|}{$\begin{array}{l}\text { Absolute substrate } \\
\text { use }\left(\mathrm{g} \cdot \mathrm{min}^{-1}\right)\end{array}$} & \multicolumn{2}{|l|}{$\begin{array}{l}\text { Relative substrate } \\
\text { use (\%) }\end{array}$} \\
\hline & & & & & & CHO oxidation & Fat oxidation & $\mathrm{CHO}$ oxidation & Fat oxidation \\
\hline $\begin{array}{l}\text { Braun et al. } \\
(2000) \text { [11] }\end{array}$ & $\begin{array}{l}15 \\
\text { (females) }\end{array}$ & $\begin{array}{l}30 \text { min cycling } \\
\text { at } 65 \% \text { relative } \\
\mathrm{VO}_{2 \max }\end{array}$ & TA & 4300 & 10 days & $\begin{array}{l}\text { SL:1.95 } \pm 0.11 \\
C H: 1.22 \pm 0.09\end{array}$ & NM & NM & NM \\
\hline $\begin{array}{l}\text { Lundby and } \\
\text { Van Hall (2002) } \\
\text { A [22] }\end{array}$ & $\begin{array}{l}8 \\
(\text { male }=6, \\
\text { female }=2)\end{array}$ & $\begin{array}{l}60 \text { min cycling a } \\
\text { t } 50 \% \text { relative } \\
\mathrm{VO}_{2 \max }\end{array}$ & $\mathrm{NH}$ & 4100 & $70 \min$ & $\begin{array}{l}\mathrm{SL}: 2.00 \pm 0.20 \\
\mathrm{AH}: 1.70 \pm 0.10\end{array}$ & $\begin{array}{l}\text { SL: } 0.30 \pm 0.01 \\
\mathrm{AH}: 0.20 \pm 0.02\end{array}$ & $\begin{array}{l}\mathrm{SL}: 73.90 \pm 2.00 \\
\mathrm{AH}: 75.50 \pm 1.90\end{array}$ & $\begin{array}{l}S L: 26.10 \pm 2.00 \\
A H: 24.50 \pm 1.90\end{array}$ \\
\hline $\begin{array}{l}\text { Lundby and } \\
\text { Van Hall (2002) } \\
\text { B [22] }\end{array}$ & $\begin{array}{l}8 \\
(\text { male }=6, \\
\text { female }=2)\end{array}$ & $\begin{array}{l}60 \text { min cycling } \\
\text { at } 50 \% \text { relative } \\
\mathrm{VO}_{2 \max }\end{array}$ & TA & 4100 & 10 days & $\begin{array}{l}\mathrm{SL}: 2.00 \pm 0.20 \\
\mathrm{CH}: 1.70 \pm 0.02\end{array}$ & $\begin{array}{l}\text { SL: } 0.30 \pm 0.01 \\
C H: 0.30 \pm 0.02\end{array}$ & $\begin{array}{l}\text { SL: } 73.90 \pm 2.00 \\
C H: 74.40 \pm 1.50\end{array}$ & $\begin{array}{l}\text { SL: } 26.10 \pm 2.00 \\
C H: 25.60 \pm 1.50\end{array}$ \\
\hline $\begin{array}{l}\text { Matu et al. } \\
\text { (2017) A [6] }\end{array}$ & 12 (males) & $\begin{array}{l}60 \text { min walking } \\
\text { at } 50 \% \text { relative } \\
\mathrm{VO}_{2 \max }\end{array}$ & $\mathrm{NH}$ & 2150 & $5 \mathrm{~h}$ & $\begin{array}{l}\mathrm{SL}: 1.56 \pm 0.35 \\
\mathrm{AH}: 1.18 \pm 0.34\end{array}$ & $\begin{array}{l}\text { SL: } 0.41 \pm 0.18 \\
\text { AH: } 0.44 \pm 0.21\end{array}$ & $\begin{array}{l}\text { SL: } 62.80 \pm 13.30 \\
\mathrm{AH}: 55.10 \pm 18.90\end{array}$ & $\begin{array}{l}\mathrm{SL}: 37.20 \pm 13.30 \\
\mathrm{AH}: 44.90 \pm 18.90\end{array}$ \\
\hline $\begin{array}{l}\text { Matu et al. } \\
\text { (2017) B [6] }\end{array}$ & 12 (males) & $\begin{array}{l}60 \text { min walking } \\
\text { at } 50 \% \text { relative } \\
\mathrm{VO}_{2 \max }\end{array}$ & $\mathrm{NH}$ & 4300 & $5 \mathrm{~h}$ & $\begin{array}{l}\mathrm{SL}: 1.56 \pm 0.35 \\
\mathrm{AH}: 0.87 \pm 0.37\end{array}$ & $\begin{array}{l}\text { SL: } 0.41 \pm 0.18 \\
\text { AH: } 0.38 \pm 0.19\end{array}$ & $\begin{array}{l}\text { SL: } 62.80 \pm 13.30 \\
A H: 50.80 \pm 19.80\end{array}$ & $\begin{array}{l}\mathrm{SL}: 37.20 \pm 13.30 \\
\mathrm{AH}: 49.20 \pm 19.80\end{array}$ \\
\hline $\begin{array}{l}\text { Morishima et al. } \\
\text { (2014) A [55] }\end{array}$ & 8 (males) & $\begin{array}{l}30 \text { min cycling } \\
\text { at } 60 \% \text { relative } \\
\mathrm{VO}_{2 \max }\end{array}$ & $\mathrm{NH}$ & 2700 & $7.5 \mathrm{~h}$ & NM & NM & $\begin{array}{l}\mathrm{SL}: 60.00 \pm 7.80 \\
\mathrm{AH}: 93.70 \pm 2.50\end{array}$ & $\begin{array}{l}\mathrm{SL}: 40.00 \pm 7.80 \\
\mathrm{AH}: 6.30 \pm 2.50\end{array}$ \\
\hline $\begin{array}{l}\text { O'Hara et al. } \\
(2017) \text { B [5] }\end{array}$ & 7 (males) & $\begin{array}{l}105 \text { min cycling } \\
\text { at } \sim 74 \% \text { relative } \\
\mathrm{VO}_{2 \max }\end{array}$ & $\mathrm{HH}$ & 3375 & $155 \mathrm{~min}$ & $\begin{array}{l}\mathrm{SL}: 2.64 \pm 0.50 \\
\mathrm{AH}: 1.47 \pm 0.62\end{array}$ & $\begin{array}{l}\text { SL: } 0.38 \pm 0.19 \\
\text { AH: } 0.63 \pm 0.25\end{array}$ & $\begin{array}{l}\mathrm{SL}: 73.10 \pm 13.10 \\
\mathrm{AH}: 48.80 \pm 18.90\end{array}$ & $\begin{array}{l}\mathrm{SL}: 26.90 \pm 13.10 \\
\mathrm{AH}: 51.20 \pm 18.90\end{array}$ \\
\hline $\begin{array}{l}\text { Peronnet et al. } \\
\text { (2006) [4] }\end{array}$ & 5 (males) & $\begin{array}{l}80 \text { min cycling } \\
\text { at } 77 \% \text { relative } \\
\mathrm{VO}_{2 \max }\end{array}$ & $\mathrm{HH}$ & 4300 & $110 \mathrm{~min}$ & $\begin{array}{l}S L: 3.25 \pm 0.13 \\
A H: 2.67 \pm 0.10\end{array}$ & $\begin{array}{l}\mathrm{SL}: 0.37 \pm 0.05 \\
\mathrm{AH}: 0.10 \pm 0.03\end{array}$ & $\begin{array}{l}\mathrm{SL}: 78.10 \pm 1.80 \\
\mathrm{AH}: 92.00 \pm 2.10\end{array}$ & $\begin{array}{l}\mathrm{SL}: 21.90 \pm 1.80 \\
\mathrm{AH}: 8.00 \pm 2.10\end{array}$ \\
\hline
\end{tabular}




\section{Study name}

Beidleman et al, (2002)

Beidleman et al, (2003) A

Beidleman et al, (2003) B

Bouissou et al, (1987)

Braun et al, (2000)

Freidmann et al, (2004)

Fulco et al, (2005) A

Fulco et al, (2005) B

Fulco et al, (2005) C

Fulco et al, (2005) D

Hopkins et al, (2003) A

Hopkins et al, (2003) B

Katayama et al, (2010)

Lundby et al, (2002) A

Lundby et al, (2002) B

Maher et al, (1974) A

Maher et al, (1974) B

Matu et al, (2017) A

Matu et al, (2017) B

Messier et al, (2017)

Noordof et al, (2013) A

Noordof et al, (2013) B

Noordof et al, (2013) C

O'Hara et al, (2017) A

O'Hara et al, (2017) B

Peronnet et al, (2006)

Wyss et al, (1990)

Young et al, (1987) A

Young et al, (1987) B

Young et al, (1987) C

Young et al, (1987) D

\section{Difference in means and $95 \% \mathrm{CI}$}

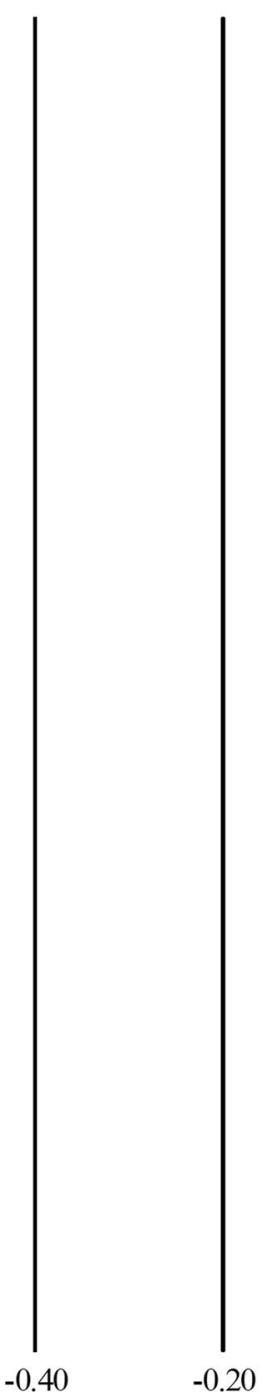

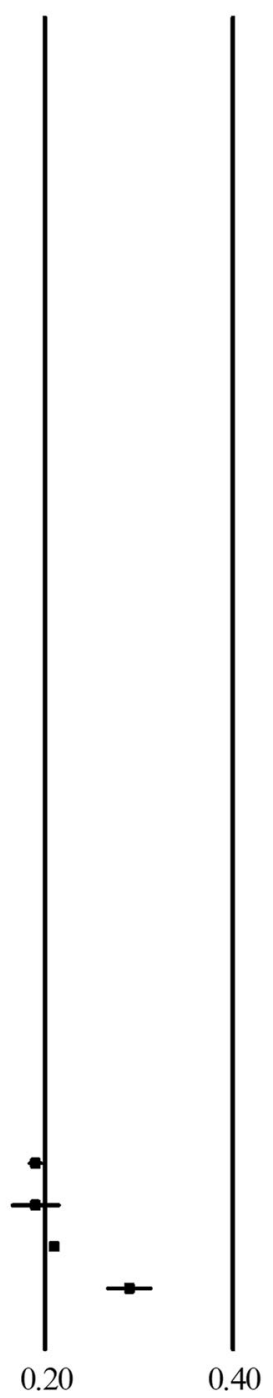

Decreased RER

Increased RER

Fig. 2 Forest plot of mean differences (means $\pm 95 \% \mathrm{Cl}$ ) for studies investigating the effects of hypoxia on RER during exercise matched for relative intensities. The size of the circle represents the relative weight of the trial. Cls are represented by a horizontal line through their representative circles. The diamond quantifies the overall mean difference (means $\pm 95 \% \mathrm{Cl}$ ). A, B, C and D refer to the different trial arms of each study. Details of which are provided in Table 1

funnel plot and Egger's regression intercept revealed little evidence of small study effects (intercept $=3.25,95 \%$ CI: -4.34 to $10.84 ; p=0.32$ ).

There was no significant change in absolute fat oxidation during exercise matched for relative intensities in hypoxia, compared with normoxia (mean difference: $-0.03 \mathrm{~g} \cdot \mathrm{min}^{-1}$, 95\% CI: -0.11 to 0.05 g. $\mathrm{min}^{-1} ; n=6, p=0.44$; Fig. 4). The degree of heterogeneity was found to be high between studies $\left(I^{2}=99.01 \%, \mathrm{Q}=506, \mathrm{\tau}^{2}=0.01, \mathrm{~d}_{\mathrm{f}}=5\right)$. Sensitivity analysis revealed minor changes only, and these changes did not substantially alter the overall mean difference.
Inspection of the funnel plot and Egger's regression intercept revealed that there was some evidence of small study effects (intercept $=-5.96,95 \% \mathrm{CI}$ : -13.14 to $1.25 ; p=0.08$ ).

\section{Risk of bias}

Since many of the studies were high altitude expeditions, certain biases were often unavoidable such as blinding of participants and personnel (Fig. 5). However, it was deemed that some of these biases could not affect the outcome variable and were therefore classified as low risk. In addition, all included studies were not clinically 
Table 3 Summary of moderator variables from the single and multiple meta-regression model for RER in response to hypoxic exposure during exercise matched for relative intensities

\begin{tabular}{|c|c|c|c|}
\hline $\begin{array}{l}\text { Moderator variable } \\
\text { RER (relative) }(n=31)\end{array}$ & $p$ value & Comparison & $\begin{array}{l}\text { Multiple regression } \\
p \text { value }\end{array}$ \\
\hline Pre-existing nutritional state & $<0.01$ & $\begin{array}{l}\text { Fasted }(n=8, \mathrm{MD}-0.07,95 \% \mathrm{Cl}-0.09 \text { to }-0.06) \\
\text { Fed }(n=18, \mathrm{MD} 0.06,95 \% \mathrm{Cl} 0.01 \text { to } 0.10)\end{array}$ & $<0.01$ \\
\hline $\begin{array}{l}\text { Carbohydrate supplementation } \\
\text { during exercise }\end{array}$ & 0.22 & $\begin{array}{l}\text { Yes }(n=4, \mathrm{MD}-0.04,95 \% \mathrm{Cl}-0.09 \text { to } 0.01) \\
\text { No }(n=27, \mathrm{MD} 0.02,95 \%-0.02 \text { to } 0.06)\end{array}$ & N/A \\
\hline Exercise mode & 0.60 & $\begin{array}{l}\text { Cycling }(n=26, \mathrm{MD} 0.02,95 \% \mathrm{Cl}-0.02 \text { to } 0.05) \\
\text { Running }(n=5, \mathrm{MD}-0.01,95 \% \mathrm{Cl}-0.04 \text { to } 0.03)\end{array}$ & N/A \\
\hline Duration of hypoxic exposure & 0.67 & $\begin{array}{l}\text { Acute }(n=22, \mathrm{MD} 0.02,95 \% \mathrm{Cl}-0.03 \text { to } 0.06) \\
\text { Chronic }(n=9, \mathrm{MD} 0.00,95 \% \mathrm{Cl}-0.03 \text { to } 0.04)\end{array}$ & N/A \\
\hline Type of hypoxia & 0.96 & $\begin{array}{l}\text { Simulated normobaric hypoxia }(n=7, \mathrm{MD} 0.01,95 \% \mathrm{Cl}-0.01 \text { to } 0.03) \\
\text { Simulated hypobaric hypoxia }(n=9, \mathrm{MD} 0.00,95 \% \mathrm{Cl}-0.03 \text { to } 0.03) \\
\text { Terrestrial altitude }(n=15, \mathrm{MD} 0.02,95 \% \mathrm{Cl}-0.03 \text { to } 0.07)\end{array}$ & N/A \\
\hline Percentage male & 0.43 & $\begin{array}{l}\text { Meta-regression percentage male vs. MD (slope } 0.0006 \text {, } \\
95 \% \mathrm{Cl}-0.0009 \text { to } 0.0021)\end{array}$ & N/A \\
\hline Exercise intensity & 0.04 & $\begin{array}{l}\text { Meta-regression of exercise intensity vs. MD (slope } 0.0033 \text {, } \\
95 \% \mathrm{Cl} 0.0002 \text { to } 0.0065 \text { ) }\end{array}$ & 0.049 \\
\hline Exercise duration & 0.78 & $\begin{array}{l}\text { Meta-regression of exercise duration vs. MD (slope }-0.0002,95 \% \\
\mathrm{Cl}-0.0018 \text { to } 0.014)\end{array}$ & N/A \\
\hline Altitude height & 0.90 & Meta-regression of altitude height vs. ES (slope $-0.00,95 \% \mathrm{Cl}-0.00$ to 0.00 ) & N/A \\
\hline
\end{tabular}

registered, therefore it is not possible to determine if all outcome variables were reported, therefore selective reporting bias was listed as unclear.

\section{Discussion}

The purpose of this systematic review and meta-analysis was to examine the effects of hypoxic exposure on substrate oxidation during exercise matched to relative intensities. There was no consistent change in relative carbohydrate or fat contribution to energy provision during exercise matched for relative intensities in hypoxia, compared with normoxia. These findings are particularly pertinent as, in contrast to exercise matched to absolute intensities, exercise matched to relative intensities isolates

\section{Study name}

Braun et al, (2000)

Lundby et al, (2002) A

Lundby et al, (2002) B

Matu et al, (2017) A

Matu et al, (2017) B

O'Hara et al, (2017) B

Peronnet et al, (2006)

\section{Difference in means and $95 \% \mathrm{CI}$}

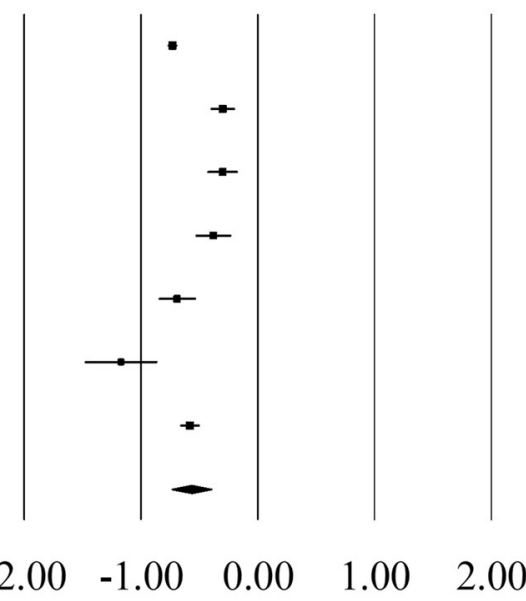

Decreased absolute carbohydrate oxidation
Increased absolute carbohydrate oxidation

Fig. 3 Forest plot of mean differences (means $\pm 95 \% \mathrm{Cl}$ ) for studies investigating the effects of hypoxia on absolute carbohydrate oxidation during exercise matched for relative intensities. The size of the circle represents the relative weight of the trial. Cls are represented by a horizontal line through their representative circles. The diamond quantifies the overall mean difference (means $\pm 95 \% \mathrm{Cl}$ ). A and B refer to the different trial arms of each study. Details of which are provided in Table 2 


\section{Study name}

Lundby et al, (2002) A

Lundby et al, (2002) B

Matu et al, (2017) A

Matu et al, (2017) B

O'Hara et al, (2017) A

Peronnet et al, (2006)

\section{Difference in means and $95 \% \mathrm{CI}$}

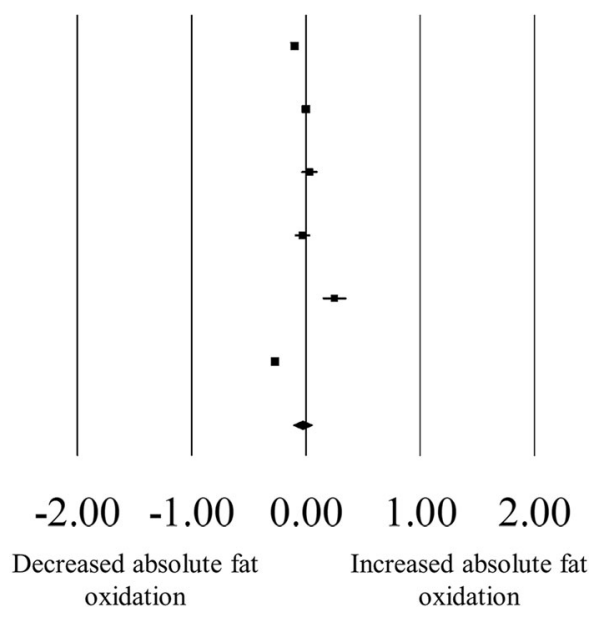

Fig. 4 Forest plot of mean differences (means $\pm 95 \% \mathrm{Cl}$ ) for studies investigating the effects of hypoxia on absolute fat oxidation during exercise matched for relative intensities. The size of the circle represents the relative weight of the trial. Cls are represented by a horizontal line through their representative circles. The diamond quantifies the overall mean difference (means $\pm 95 \% \mathrm{Cl}$ ). A and B refer to the different trial arms of each study. Details of which are provided in Table 2

the effect of hypoxia by normalising the exercise intensities between conditions [22]. Additionally, the heterogeneous findings of the current literature may be explained by a number of differing experimental characteristics, such as pre-exercise nutritional status and exercise intensity.

There was no significant change observed in RER during exercise matched for relative intensities in hypoxia, compared with normoxia. In addition, there was no significant change in relative carbohydrate or fat oxidation in the same circumstances. It was deemed that RER was the most useful outcome variable due the largest number of comparisons as a result of being the most frequently reported in the literature. As expected, reduced absolute carbohydrate rates were observed in hypoxia during exercise matched to relative intensities, due to the lower absolute workload [27] performed in hypoxia than normoxia and the subsequent reduction in energy expenditure. However, no significant change in absolute fat oxidation was observed in hypoxia, likely due to the limited changes in the contribution from this fuel source at moderate $\left(40-55 \% \mathrm{VO}_{2 \max }\right)$ exercise intensities [28].

The current review found that an increase in RER was induced during exercise matched for relative intensities in hypoxia compared with normoxia when participants were in the fed state. Alternatively, a decrease in RER was induced when in the fasted state. The increase in

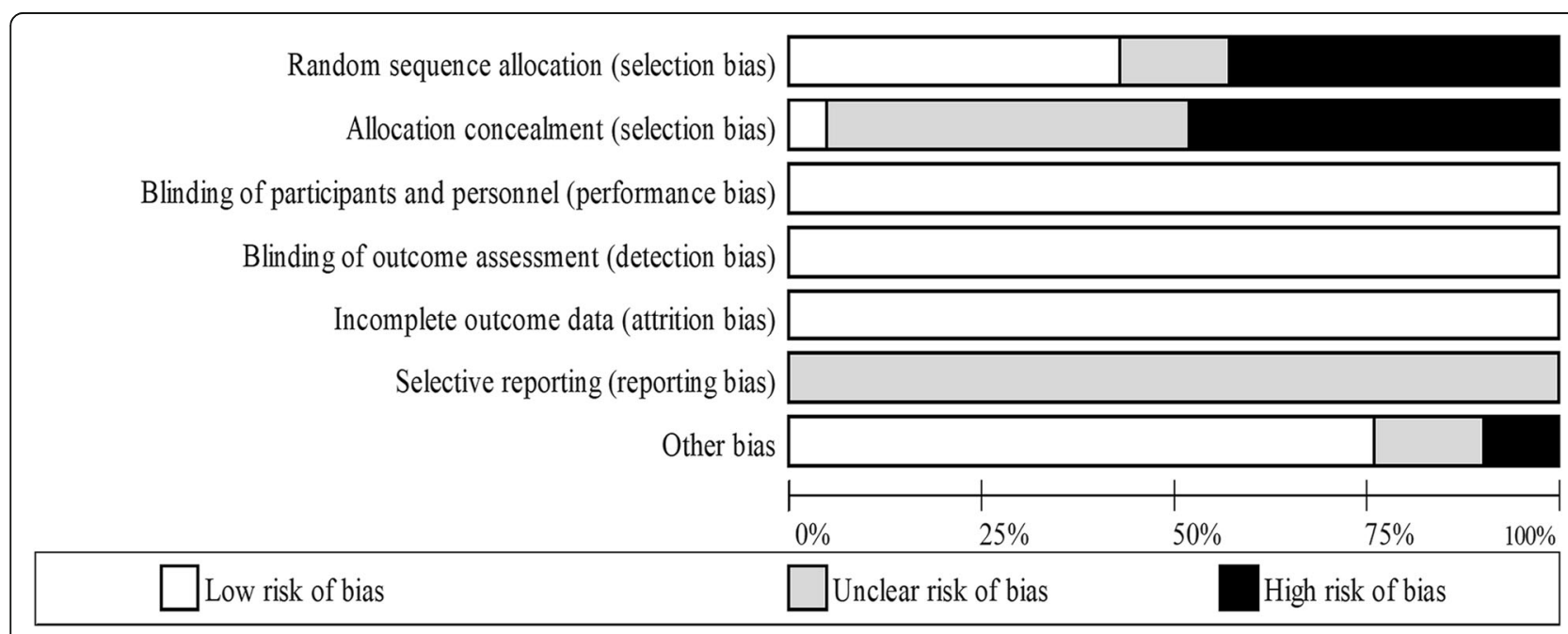

Fig. 5 Assessment of risk of bias (Cochrane's collaboration tool) 
endogenous carbohydrate stores as a result of feeding may facilitate a hypoxic-induced physiological demand for increased carbohydrate oxidation, thus potentiating the fuel shift. The mobilisation and oxidation of these stores may be augmented by the synergistic effect of feeding [29] and hypoxia [3] on sympathetic nervous system activity (i.e. increased secretion of epinephrine and norepinephrine) and resultant increases in gluconeogenesis and glycogenolysis. Interestingly, recent evidence also suggests that the rise in circulating insulin concentrations after feeding may increase carbohydrate oxidation from muscle glycogen stores, even before the ingested carbohydrate has been transported into the muscle [30]. This is supported by previous work demonstrating a reduction in muscle glycogen concentrations one-hour after consuming a mixed macronutrient meal, before increasing again in the subsequent hours [31]. This increase in insulin concentrations after a pre-exercise meal may be potentiated by hypoxia [6], thereby enhancing the inhibition of lipolysis and FFA mobilisation [32] to increase carbohydrate oxidation. In contrast, fasted exercise may elicit a decrease in RER via the enhanced activation of PPAR $\alpha$ due to both hypoxia [8] and fasting [33]. The synergistic effect of both factors may further disrupt glycolysis [9] and enable greater fat flux [10].

In addition, an increased RER was observed during exercise matched to relative intensities in hypoxia, compared with normoxia, during exercise performed at higher intensities. This effect may be mediated by the hypoxic effect of altitude and high intensity exercise, augmenting skeletal muscle hypoxia [34]. The mechanisms associated with these changes are likely explained as per the physiological response to increased exercise intensities in normoxic environments. In this regard, higher exercise intensities induce a reduction in adipose tissue blood flow, which may attenuate the release of FFA resulting in decreased delivery to the contracting muscle [35]. Further, greater exercise intensities stimulate greater flux through the glycolytic pathway and pyruvate dehydrogenase complex (PDC) than flux through the tricarboxylic acid cycle, resulting in the accumulation of acetyl coA [36]. The subsequent acylation of the carnitine pool has been suggested to result in a marked decrease in muscle free carnitine and downregulation of carnitine palmitoyltransferase I (CPT-1), the enzyme responsible for transporting long chain fatty acids into the mitochondrial matrix [28]. Alternatively, the effect of hypoxia on the sympathetic nervous system may be potentiated by greater exercise intensities, enabling greater carbohydrate oxidation due to increased glycogenolysis, a result of enhanced glycogen phosphorylase activity, sarcoplasmic $\mathrm{Ca}^{2+}$, inorganic phospohate and cyclic AMP $[37,38]$. Numerous mechanisms are proposed to explain the reduction in FFA oxidation with increasing exercise intensities [39], however detailed discussion of all theories is out of the scope of this review.

The large between study heterogeneity in relation to RER during exercise matched to relative intensities was explained in part by pre-exercise nutritional state and exercise intensity $(\sim 42 \%)$. The remaining, unexplained heterogeneity may highlight some limitations of the present meta-analysis. Results from a meta-regression are indicative of a between-study relationship, however due to confounding bias (i.e. one experimental characteristic may reflect a true association with other correlated, known or unknown characteristics), this relationship may not be replicated within-studies. This is termed aggregation bias. As such, moderator analysis should be regarded as hypothesis gathering, rather than hypothesis testing [40]. These moderators should therefore subsequently be investigated using a within-measures design via randomised controlled trials, generating causal, rather than observational relationships. Further, the unexplained heterogeneity may be due to methodological heterogeneity (i.e. study quality/measurement error) or insufficient trials to generate the appropriate power to fully explain the heterogeneity. A greater quantity and quality of research regarding substrate oxidation during hypoxia would help to further explain the heterogeneity between trials Further research is required to confirm the findings from this meta-analysis and quantify the influence of the fasted and fed state and exercise intensity on substrate utilisation in hypoxia.

The present meta-analysis provides clarity, and therefore facilitates an accurate interpretation, of the current literature. These findings may inform nutritional strategies for mountaineers, military personnel and athletes during exposure to altitude, subsequently limiting the detrimental exercise performance experienced in such conditions. The performance benefits of maintaining exogenous carbohydrate oxidation and/or endogenous carbohydrate stores via pre-exercise carbohydrate consumption in normoxia are well documented [41]. As such, findings from this review suggest that a physiological drive for carbohydrate oxidation in hypoxia may be facilitated by an increased carbohydrate intake prior to exercise, in order to avoid an accelerated depletion of muscle glycogen, and shift back to the less efficient oxidation of fat [35]. In contrast, the use of low carbohydrate intake strategies to enhance endurance training metabolic adaptations is growing in popularity [42] and findings from the present meta-analysis may have implications for such strategies. Specifically, the combined effect of training in hypoxia in a glycogen depleted state may potentiate the metabolic adaptations of 'training low'. Alternatively, a number of studies have demonstrated that changes in substrate utilisation during exercise in hypoxia may have implications for metabolic disease programmes $[43,44]$. As such, the clinical translation of the current study warrants further investigation. 
Despite the important findings observed in the current meta-analysis, some notable limitations must be acknowledged. First, the equivocal findings observed in RER and relative substrate oxidation reflects the heterogeneity in the literature, rather than an absence of change in substrate oxidation due to hypoxic exposure. This heterogeneity is likely due to differing experimental characteristics between studies and although moderator analysis was employed to identify these factors, these findings should be interpreted with caution. Second, the physiological determinants of substrate oxidation (e.g. hormonal factors), were not quantified and therefore physiological mechanisms were difficult to elucidate. This was, however, beyond the scope of this study. Finally, despite an extensive search returning 1743 records, we cannot guarantee that our search was completely exhaustive of the relevant literature. However, should the primary or secondary aim of a study be related to this area, they are likely to have been detected in our search.

\section{Conclusions}

This meta-analysis did not demonstrate a consistent change in relative carbohydrate or fat contribution to energy provision during exercise matched for relative intensities in hypoxia, compared with normoxia. These findings reflect the heterogeneity in the current literature. A metabolically efficient shift to carbohydrate oxidation may be induced by consumption of a pre-exercise meal and a higher exercise intensity. A significant amount of between-study heterogeneity could not be explained by the moderators used in this meta-analysis, highlighting the need for future research to further investigate moderators of this effect in a randomised and controlled fashion.

\section{Additional files}

Additional file 1: Search strategy and key words. (DOCX 13 kb)

Additional file 2: Individual study statistics for studies evaluating RER during exercise matched for relative intensities in hypoxia compared with normoxia. A, B, C and D refer to the different trial arms of each study. Details of which are provided in Table 1. (DOCX $26 \mathrm{~kb}$ )

Additional file 3: Individual study statistics for studies evaluating relative carbohydrate oxidation during exercise matched for relative intensities in hypoxia compared with normoxia. A and B refer to the different trial arms of each study. Details of which are provided in Table 2. (DOCX 15 kb)

Additional file 4: Individual study statistics for studies evaluating relative fat oxidation during exercise matched for relative intensities in hypoxia compared with normoxia. A and $B$ refer to the different trial arms of each study. Details of which are provided in Table 2. (DOCX $15 \mathrm{~kb}$ )

Additional file 5: Individual study statistics for studies evaluating absolute carbohydrate oxidation during exercise matched for relative intensities in hypoxia compared with normoxia. A and B refer to the different trial arms of each study. Details of which are provided in Table 2. (DOCX $15 \mathrm{~kb}$ )

Additional file 6: Individual study statistics for studies evaluating absolute fat oxidation during exercise matched for relative intensities in hypoxia compared with normoxia. A and B refer to the different trial arms of each study. Details of which are provided in Table 2. (DOCX $15 \mathrm{~kb}$ )
Additional file 7: Forest plot of mean differences (means $\pm 95 \% \mathrm{Cl}$ ) for studies investigating relative carbohydrate oxidation during exercise matched for relative intensities in hypoxia compared with normoxia. The size of the circle represents the relative weight of the trial. Cls are represented by a horizontal line through their representative circles. The diamond quantifies the overall mean difference (means $\pm 95 \% \mathrm{Cl}$ ). A and $B$ refer to the different trial arms of each study. Details of which are provided in Table 2. (TIF $381 \mathrm{~kb}$ )

Additional file 8: Forest plot of mean differences (means $\pm 95 \% \mathrm{Cl}$ ) for studies investigating relative fat oxidation during exercise matched for relative intensities in hypoxia compared with normoxia. The size of the circle represents the relative weight of the trial. Cls are represented by a horizontal line through their representative circles. The diamond quantifies the overall mean difference (means $\pm 95 \% \mathrm{Cl}$ ). A and B refer to the different trial arms of each study. Details of which are provided in Table 2. (TIF $436 \mathrm{~kb}$ )

\section{Abbreviations}

$\mathrm{AH}$ : Acute hypoxia; $\mathrm{CH}$ : Chronic hypoxia; $\mathrm{CHO}$ : Carbohydrate; $\mathrm{Cl}$ : Confidence interval; CPT-1: Carnitine palmitoyltransferase; D1: mean of group 1 - mean of total group; D2: mean of group 2 - mean of total group; FFA: Free fatty acid; HH: Hypobaric hypoxia; HIF-1 a: Hypoxic-inducible factor 1 alpha; MD: Mean difference; $n^{1}$ : Sample size of group $1 ; n^{2}$ : Sample size of group 2; $\mathrm{NH}$ : Normobaric hypoxia; NM: Not measured; PPARa: Peroxisome proliferatoractivated receptor alpha; PRISMA: Preferred Reporting Items for Systematic Review and Meta-analyses; RER: Respiratory exchange ratio; S1: Standard deviation of group 1; S2: Standard deviation of group 2; SD: Standard deviation; SL: Sea level; TA: Terrestrial altitude

\section{Acknowledgements}

Not applicable.

\section{Funding}

Not applicable.

\section{Availability of data and materials}

All data generated and analysed in this study are included in this published article and the associated supplementary files.

\section{Authors' contributions}

AG, JOH, KD and RK conceived and planned the study. AG, JM, OS and KD conducted the search and were involved in the review and extraction of all data. AG, JM and KD performed the analysis on the extracted data. All authors discussed the interpretation of results. AG wrote the manuscript. All authors provided critical feedback and helped shape the manuscript. All authors read and approved the final manuscript.

Ethics approval and consent to participate Not applicable.

Consent for publication

Not applicable.

Competing interests

The authors declare that they have no competing interests.

\section{Publisher's Note}

Springer Nature remains neutral with regard to jurisdictional claims in published maps and institutional affiliations.

\section{Author details}

${ }^{1}$ Institute for Sport, Physical Activity and Leisure, Leeds Beckett University, Leeds LS6 3QS, UK. 'Human Nutrition Research Centre, Institute of Cellular Medicine, Newcastle University, Leech Building, Framlington Place, Newcastle Upon Tyne NE2 4HH, UK. ${ }^{3}$ Leeds Institute of Rheumatic and Musculoskeletal Medicine, 2nd floor Chapel Allerton Hospital, Chapeltown Road, Leeds LS7 4SA, UK. 


\section{Received: 19 October 2018 Accepted: 13 February 2019}

\section{Published online: 27 February 2019}

\section{References}

1. Brocherie F, Girard O, Millet G. Emerging environmental and weather challenges in outdoor sports. Climate. 2015:3:492-521.

2. Deb S, Brown D, Gough L, Mclellan C, Swinton P, Sparks A, Mcnaughton L. Quantifying the effects of acute hypoxic exposure on exercise performance and capacity: a systematic review and meta-regression. Eur J Sport Sci. 2018; 18:243-56.

3. Katayama K, Goto K, Ishida K, Ogita F. Substrate utilization during exercise and recovery at moderate altitude. Metabolism. 2010;59:959-66.

4. Péronnet F, Massicotte D, Folch N, Melin B, Koulmann N, Jimenez C, Bourdon L, Launay JC, Savourey G. Substrate utilization during prolonged exercise with ingestion of 13C-glucose in acute hypobaric hypoxia 4,300 m). Eur J Appl Physiol. 2006;97:527-34.

5. O'Hara JP, Woods DR, Mellor A, Boos C, Gallagher L, Tsakirides C, Arjomandkhah NC, Holdsworth DA, Cooke CB, Morrison DJ, Preston T, King R. A comparison of substrate oxidation during prolonged exercise in men at terrestrial altitude and normobaric normoxia following the coingestion of 13C glucose and 13C fructose. Physiol Rep. 2017. https://doi.org/10.14814/ phy2.13101.

6. Matu J, Deighton K, Ispoglou T, Duckworth L. The effect of moderate versus severe simulated altitude on appetite, gut hormones, energy intake and substrate oxidation in men. Appetite. 2017;113:284-92.

7. Brooks G, Butterfield G, Wolfe R, Groves B, Mazzeo R, Sutton J, Reeves J. Increased dependance on blood glucose after acclimatization to $4300 \mathrm{~m}$. J Appl Physiol. 1991;70:919-27.

8. Aragones J, Schneider M, Van Geyte K, Fraisl P, Dresselaers T, Mazzone M, Dirkx R, Zacchigna S, Lemieux $H$, Jeoung NH, et al. Deficiency or inhibition of oxygen sensor Phd1 induces hypoxia tolerance by reprogramming basal metabolism. Nat Genet. 2008;40:170-80.

9. Huang B, Wu P, Bowker-Kinley M, Harris R. Regulation of pyruvate dehydrogenase kinase expression by peroxisome proliferator-activated receptor-alpha ligands, glucocorticoids, and insulin. Diabetes. 2002;51:276-83.

10. Spriet $L$, Watt $M$. Regulatory mechanisms in the interaction between $\mathrm{CHO}$ and lipid oxidation during exercise. Acta Physiol Scand. 2003;178:443-52.

11. Braun B, Mawson J, Muza SR, Dominick S, Brookes G, Horning M, Rock PB, Moore L, Mazzeo R, Ezeji-Okoye S, Butterfield G. Women at altitude. Carbohydrate utilisation during exercise at 4300m. J Appl Physiol. 2000;88: 246-56.

12. Savourey G, Launay JC, Besnard Y, Guinet A, Travers S. Normo- and hypobaric hypoxia: are there any physiological differences? Eur J Appl Physiol. 2003;89:122-6.

13. Saugy J, Schmitt L, Hauser A, Constantin G, Cejuela R, Faiss R, Wehrlin J, Rosset J, Robinson N, Millet G. Same performance changes after live hightrain low in Normobaric vs. Hypobaric Hypoxia. Front Physiol. 2016;7.

14. Kammerer K, Faihs V, Hulde N, Bayer A, Hübner M, Brettner F, Karlen W, Kröpfl J, Rehm M, Spengler C, Schäfer S. Changes of hemodynamic and cerebral oxygenation after exercise in normobaric and hypobaric hypoxia: associations with acute mountain sickness. Ann Occup Environ Med. 2018;30.

15. Hochachka PW, Stanley C, Matheson GO, McKenzie DC, Allen PS, Parkhouse WS. Metabolic and work efficiencies during exercise in Andean natives. J Appl Physiol. 1991;70:1720-30.

16. Beidleman BA, Rock PB, Muza SR, Fulco CS, Gibson LL, Kamimori GH, Cymerman A. Substrate oxidation is altered in women during exercise upon acute altitude exposure. Med Sci Sports Exerc. 2002;34:430-7.

17. Blaak E. Gender differences in fat metabolism. Curr Opin Clin Nutr Metab Care. 2001:4:499-502.

18. Tarnopolsky MA, Rennie CD, Robertshaw HA, Fedak-Tarnopolsky SN, Devries MC, Hamadeh MJ. Influence of endurance exercise training and sex on intramyocellular lipid and mitochondrial ultrastructure, substrate use, and mitochondrial enzyme activity. Am J Physiol Regul Integr Comp Physiol. 2007;292:R1271-8.

19. Devries MC, Lowther SA, Glover AW, Hamadeh MJ, Tarnopolsky MA. IMCL area density, but not IMCL utilization, is higher in women during moderateintensity endurance exercise, compared with men. Am J Physiol Regul Integr Comp Physiol. 2007;293:R2336-42.

20. D'eon T, Sharoff C, Chipkin SR, Grow D, Ruby BC, Braun B. Regulation of exercise carbohydrate metabolism by estrogen and progesterone in women. Am J Physiol Endocrinol Metab. 2002;283:E1046-55.
21. Campbell SE, Angus DJ, Febbraio MA. Glucose kinetics and exercise performance during phases of the menstrual cycle: effect of glucose ingestion. Am J Physiol Endocrinol Metab. 2001;281:814-25.

22. Lundby C, Van Hall G. Substrate utilization in sea level residents during exercise in acute hypoxia and after 4 weeks of acclimatization to $4100 \mathrm{~m}$. Acta Physiol Scand. 2002;176:195-201.

23. Liberati A, Altman DG, Tetzlaff J, Mulrow C, Gotzsche PC, loannidis JP, Clarke M, Devereaux PJ, Kleijnen J, Moher D. The PRISMA statement for reporting systematic reviews and meta-analyses of studies that evaluate healthcare interventions: explanation and elaboration. BMJ. 2009;339:b2700.

24. Bartsch P, Saltin B. General introduction to altitude adaptation and mountain sickness. Scand J Med Sci Sports. 2008;18:1-10.

25. Poole DC, Jones AM. Oxygen uptake kinetics. Compr Physiol. 2012. https:// doi.org/10.1002/cphy.c100072.

26. Higgins JP, Green S. Cochrane handbook for systematic reviews of interventions: The Cochrane Collaboration; 2011.

27. Romijn JA, Coyle EF, Sidossis LS, Gastaldelli A, Horowitz JF, Endert E, Wolfe RR. Regulation of endogenous fat and carbohydrate metabolism in relation to exercise intensity and duration. Am J Physiol Endocrinol Metab. 1993; 265(Suppl 3):E380-91.

28. Van Loon L, Greenhaff P, Constantin-Teodosiu D, Saris W, Wagenmakers A. The effect of increasing exercise intensity on muscle fuel utilisation in humans. J Physiol. 2001;536:295-304.

29. Tentolouris N, Tsigos C, Perea D, Koukou E, Kyriaki D, Kitsou E, Daskas S, Daifotis Z, Makrilakis K, Raptis SA, Katsilambros N. Differential effects of highfat and high-carbohydrate isoenergetic meals on cardiac autonomic nervous system activity in lean and obese women. Metabolism. 2003;52: 1426-32.

30. Edinburgh RM, Hengist A, Smith HA, Travers RL, Koumanov F, Betts JA, Thompson D, Walhin JP, Wallis GA, Hamilton DL, Stevenson EJ, Tipton KD, Gonzalez JT. Pre-exercise breakfast ingestion versus extended overnight fasting increases postprandial glucose flux after exercise in healthy men. Am J Physiol Endocrinol Metab. 2018. https://doi.org/10.1152/ajpendo. 00163.2018.

31. Taylor R, Price TB, Katz LD, Shulman RG, Shulman Gl. Direct measurement of change in muscle glycogen concentration after a mixed meal in normal subjects. Am J Physiol Endocrinol Metab. 1993;265:E224-9.

32. Coyle E, Jeukendrup A, Wagenmakers A, Saris W. Fatty acid oxidation is directly regulated by carbohydrate metabolism during exercise. Am J Physiol Endocrinol Metab. 1997;273(Suppl 2):E268-73.

33. Van Diepen J, Jansen P, Ballak D, Hijmans A, Hooiveld G, Rommelaere S, Galland F, Naquet P, Schrauwen P. PPAR-alpha dependent regulation of vanin-1 mediates hepatic lipid metabolism. J Hepatol. 2014;61:366-72.

34. Mazzeo RS. Physiological responses to exercise at altitude : an update. Sports Med (Auckland, NZ). 2008;38:1-8.

35. Romijn J, Coyle E, Sidossis L, Zhang X, Wolfe R. Relationship between fatty acid delivery and fatty acid oxidation during strenuous exercise. J Appl Phyiol. 1995. https://doi.org/10.1152/jappl.1995.79.6.1939.

36. Sahlin K. Muscle carnitine metabolism during incremental dynamic exercise in humans. Acta Physiol Scand. 1990. https://doi.org/10.1111/j.1748-1716. 1990.tb08845.x.

37. Howlett R, Parolin M, Dyck D, Hultman E, Jones N, Heigenhauser G, Spriet L. Regulation of skeletal muscle glycogen phosphorylase and PDH at varying exercise outputs. Am J Physiol Regul Integr Comp Physiol. 1998;275(Suppl 2):R418-25.

38. Watt M, Howlett K, Febbraio M, Spriet L, Hargreaves M. Adrenaline increases skeletal muscle glycogenolysis, $\mathrm{PDH}$ activation and carbohydrate oxidation during moderate exercise in humans. J Physiol. 2001;534:269-78.

39. Spriet LL. New insights into the interaction of carbohydrate and fat metabolism during exercise. Sports Med. 2014;44(Suppl 1):S87-96.

40. Thompson S, Higgins JP. How should meta-regression analyses be undertaken and interpreted? Stat Med. 2002;21:1559-73.

41. Ormsbee MJ, Bach CW, Baur DA. Pre-exercise nutrition: the role of macronutrients, modified starches and supplements on metabolism and endurance performance. Nutrients. 2014;6:1782-808.

42. Impey S, Hearris M, Hammond K, Bartlett J, Louis J, Close G, Morton J. Fuel for the work required: a theoretical framework for carbohydrate periodization and the glycogen threshold hypothesis. Sports Med. 2018;48:1031-48.

43. Bailey DM, Davies B, Baker J. Training in hypoxia: modulation of metabolic and cardiovascular risk factors in men. Med Sci Sports Exerc. 2000;32:1058-66. 
44. Haufe S, Wiesner S, Engeli S, Luft FC, Jordan J. Influences of normobaric hypoxia training on metabolic risk markers in human subjects. Med Sci Sports Exerc. 2008;40:1939-44.

45. Beidleman B, Muza S, Fulco C, Cymerman A, Ditzler D, Stulz D, Staab J, Robinson S, Skrinar G, Lewis S, Sawka M. Intermittent altitude exposures improve muscular performance at 4300m. J Appl Phyiol. 2003;95:1824-32.

46. Bouissou P, Guezennec Y, Defer G, Pesquies P. Oxygen consumption, lactate accumulation and sympathetic response during prolonged exercise under hypoxia. Int J Sports Med. 1987;8:266-9.

47. Friedmann B, Bauer T, Menold E, Bartsch P. Exercise with the intensity of the individual anaerobic threshold in acute hypoxia. Med Sci Sports Exerc. 2004; 36:1737-42.

48. Fulco CS, Kambis KW, Friedlander AL, Rock PB, Muza SR, Cymerman A. Carbohydrate supplementation improves time-trial cycle performance during energy deficit at 4,300-m altitude. J Appl Physiol. 2005:99:867-76.

49. Hopkins S, Bogaard H, Niizeki K, Yamaya Y, Ziegler M, Wagner P. Betaadrenergic or parasympathetic inhibition, heart rate and cardiac output during normoxic and acute hypoxic exercise in humans. J Physiol. 2003;550: 605-16.

50. Maher J, Jones L, Hartley H. Effect of high altitude exposure on sub-maximal endurance capacity of men. J Appl Physiol. 1974;37:895-8.

51. Messier FM, Le Moyec L, Santi C, Gaston AF, Triba MN, Roca E, Durand F, The impact of moderate altitude on exercise metabolism in recreational sportsmen: a nuclear magnetic resonance metabolomic approach. Appl Physiol Nutr Metab. 2017:42:1135-41.

52. Noordhof DA, Schoots T, Hoekert DH, De Koning JJ. Is gross efficiency lower at acute simulated altitude than at sea level? Int I Sports Physiol. 2013;8: 319-22.

53. Wyss V, Ganzit GP, Rienzi A. Effects of L-carnitine administration on Vo2max and the aerobic-anaerobic threshold in normoxia and acute hypoxia. Eur J Appl Physiol Occup Physiol. 1990;60:1-6.

54. Young PM, Rock PB, Fulco CS, Trad LA, Forte VA Jr, Cymerman A. Altitude acclimatization attenuates plasma ammonia accumulation during submaximal exercise. J Appl Physiol. 1987;63:758-64.

55. Morishima T, Mori A, Sasaki H, Goto K. Impact of exercise and moderate hypoxia on glycemic regulation and substrate oxidation pattern. PLoS One. 2014;9:e108629.

Ready to submit your research? Choose BMC and benefit from:

- fast, convenient online submission

- thorough peer review by experienced researchers in your field

- rapid publication on acceptance

- support for research data, including large and complex data types

- gold Open Access which fosters wider collaboration and increased citations

- maximum visibility for your research: over $100 \mathrm{M}$ website views per year

At $\mathrm{BMC}$, research is always in progress.

Learn more biomedcentral.com/submissions 